\title{
Effect of total flavonoids from Scutellaria baicalensis on dopaminergic neurons in the substantia nigra
}

\author{
XUE-LI LI ${ }^{1}$, XIAO-FAN XU ${ }^{1 *}$, QING-XIA BU ${ }^{1 *}$, WEI-RONG JIN ${ }^{1}$, \\ QIAN-RU SUN ${ }^{2}$, DE-PENG FENG ${ }^{1}$, QING-JV ZHANG ${ }^{1}$ and LE-XIN WANG ${ }^{3}$

\begin{abstract}
Departments of ${ }^{1}$ Neurology and ${ }^{2}$ Neuroimmune Laboratory, Liaocheng People's Hospital and Liaocheng Clinical School of Taishan Medical University, Liaocheng, Shandong 252000, P.R. China;

${ }^{3}$ School of Biomedical Sciences, Charles Sturt University, Wagga Wagga, NSW 2678, Australia
\end{abstract}

Received March 28, 2016; Accepted June 22, 2016

DOI: $10.3892 /$ br.2016.713

\begin{abstract}
The aim of the present study was to investigate the effect of Scutellaria baicalensis stem-leaf total flavonoid (SSTF) on the dopaminergic neurons in the substantia nigra in a mouse model of Parkinson's disease (PD). The mouse model was established by intravenous injection of 1-methyl-4-phenyl-1,2,3,6-tetrahydropyridine (MPTP). SSTF $(5 \mathrm{mg} / \mathrm{kg}$ ) was administered to the mice before or after MPTP injection, and the effects of SSTF on the behavior of the mice and the dopaminergic neurons in the substantia nigra were assessed. In addition, the level of serum malondialdehyde (MDA) was measured. Following injection of MPTP, the number of dopaminergic neurons in the substantia nigra was decreased and the neurons appeared atrophic. In addition, the level of serum MDA in the MPTP mice increased. The mean behavioral scores and the number of dopaminergic neurons in the SSTF treatment groups were significantly higher than in the MPTP group $(\mathrm{P}<0.05)$, and the mean serum MDA levels were significantly lower $(\mathrm{P}<0.05)$. Thus, SSTF improves the behaviors and the numbers of dopaminergic neurons in the substantia nigra in MPTP-induced PD in mice. These beneficial effects appear to be associated with the reduction in serum MDA.
\end{abstract}

\section{Introduction}

Parkinson's disease (PD) is a chronic and progressive neurological disorder, which is associated with significant morbidity and mortality (1). The primary therapeutic strategy for PD is

Correspondence to: Professor Le-Xin Wang, School of Biomedical Sciences, Charles Sturt University, Boorooma Street, Wagga Wagga, NSW 2678, Australia

E-mail: 1wang@csu.edu.au

*Contributed equally

Key words: flavonoid, Parkinson's disease, dopaminergic neurons, oxidative stress, Scutellaria baicalensis the administration of therapeutic agents, such as levodopa (1). However, higher doses and long-term use of levodopa are associated with adverse effects, such as motor fluctuations and dyskinesia (1). Early treatment of PD with other agents, such as dopamine agonists and monoamine oxidase type B inhibitors, provides symptomatic benefits and delays the initiation of levodopa therapy (1). The development of novel therapeutic agents for PD that have improved therapeutic efficacy and fewer side effects than current anti-PD medications is, therefore, being actively investigated.

Scutellaria baicalensis stem-leaf total flavonoid (SSTFs) has been identified as neuro-protective on central nervous system (2). However, the underlying mechanisms of the neuroprotective effect of SSTF has not been well-delineated. The present study was designed to assess the effects and mechanisms of SSTF to dopaminergic neurons in the substantia nigra compact (SNC) of a mouse model of 1-methyl-4-phenyl-1,2,3,6-tetrahydropyridine (MPTP)-induced PD (3).

\section{Materials and methods}

Animal model. The C57BL/6J male mice (age, 8-10 weeks; weight, 20-25 g) were purchased from the experimental animal center of the Academy of Military Medical Sciences (Beijing, China). They were maintained under a 12-h dark/light cycle under standard laboratory conditions with free access to food and water. The study was approved by the animal ethics committee of Liaocheng People's Hospital (Liaocheng, China).

Thirty-two mice were randomly divided into four groups of eight per group: Control group, received no treatment; MPTP group, treated with one dose of intravenous MPTP $(25 \mathrm{mg} / \mathrm{kg})$, followed by daily intravenous injection of normal saline for 5 days; SSTF + MPTP group, daily intravenous injection of SSTF $(5 \mathrm{mg} / \mathrm{kg}$ ) for 5 days followed by one dose of MPTP treatment at $25 \mathrm{mg} / \mathrm{kg}$; MPTP + SSTF group, treated with one dose of MPTP $(25 \mathrm{mg} / \mathrm{kg})$, followed by daily SSTF $(5 \mathrm{mg} / \mathrm{kg})$ for 5 days.

Open-field test evaluation. The open-field test was used to monitor the behaviors of mice for 3-5 min after MPTP injection. Mice were suspended on a string (diameter, $3 \mathrm{~mm}) 30 \mathrm{~cm}$ 
Table I. Behavior scores.

\begin{tabular}{|c|c|c|c|c|c|c|}
\hline Group & Day 4 & Day 5 & Day 6 & Day 7 & Day 8 & Day 9 \\
\hline Control & $0.00 \pm 0.00$ & $0.00 \pm 0.00$ & $0.00 \pm 0.00$ & $0.00 \pm 0.00$ & $0.00 \pm 0.00$ & $0.00 \pm 0.00$ \\
\hline МРТР & $1.88 \pm 0.35^{\mathrm{a}}$ & $2.13 \pm 0.35^{\mathrm{a}}$ & $2.25 \pm 0.46$ & $2.34 \pm 0.52^{\mathrm{a}}$ & $2.50 \pm 0.53^{\mathrm{a}}$ & $2.63 \pm 0.52^{\mathrm{a}}$ \\
\hline $\mathrm{MPTP}+\mathrm{SSTF}$ & $1.13 \pm 0.35^{\mathrm{a}, \mathrm{b}}$ & $1.38 \pm 0.52^{\mathrm{a}, \mathrm{b}}$ & $1.50 \pm 0.53^{\mathrm{a}, \mathrm{b}}$ & $1.50 \pm 0.53^{\mathrm{a}, \mathrm{b}}$ & $1.63 \pm 0.52^{\mathrm{a}, \mathrm{b}}$ & $1.50 \pm 0.76^{\mathrm{a}, \mathrm{b}}$ \\
\hline SSTF + MPTP & $1.00 \pm 0.00^{\mathrm{a}, \mathrm{b}}$ & $1.25 \pm 0.46^{\mathrm{a}, \mathrm{b}}$ & $1.38 \pm 0.52^{\mathrm{a}, \mathrm{b}}$ & $1.38 \pm 0.52^{\mathrm{a}, \mathrm{b}}$ & $1.50 \pm 0.53^{\mathrm{a}, \mathrm{b}}$ & $1.38 \pm 0.74^{\mathrm{a}, \mathrm{b}}$ \\
\hline
\end{tabular}

${ }^{\mathrm{a}} \mathrm{P}<0.05$ vs. control group; ${ }^{\mathrm{b}} \mathrm{P}<0.05$ vs. MPTP group. MPTP, 1-methyl-4-phenyl-1,2,3,6-tetrahydropyridine; SSTF, Scutellaria baicalensis stem-leaf total flavonoid. Values are presented as means \pm standard deviations.

Table II. Hanging test scores.

\begin{tabular}{|c|c|c|c|c|c|c|}
\hline Group & Day 4 & Day 5 & Day 6 & Day 7 & Day 8 & Day 9 \\
\hline Control & $6.00 \pm 0.00$ & $6.00 \pm 0.00$ & $6.00 \pm 0.00$ & $6.00 \pm 0.00$ & $6.00 \pm 0.00$ & $6.00 \pm 0.00$ \\
\hline MPTP & $1.88 \pm 0.35^{\mathrm{a}}$ & $1.75 \pm 0.46^{\mathrm{a}}$ & $1.63 \pm 0.52$ & $1.63 \pm 0.52^{\mathrm{a}}$ & $1.38 \pm 0.52^{\mathrm{a}}$ & $1.25 \pm 0.46^{\mathrm{a}}$ \\
\hline MPTP + SSTF & $4.25 \pm 0.71^{\mathrm{a}, \mathrm{b}}$ & $4.13 \pm 0.64^{\mathrm{a}, \mathrm{b}}$ & $4.00 \pm 0.53^{\mathrm{a}, \mathrm{b}}$ & $3.88 \pm 0.35^{\mathrm{a}, \mathrm{b}}$ & $4.00 \pm 0.00^{\mathrm{a}, \mathrm{b}}$ & $3.63 \pm 0.74^{\mathrm{a}, \mathrm{b}}$ \\
\hline SSTF + MPTP & $4.38 \pm 0.74^{\mathrm{a}-\mathrm{c}}$ & $4.13 \pm 0.64^{\mathrm{a}-\mathrm{c}}$ & $4.13 \pm 0.64^{\mathrm{a}-\mathrm{c}}$ & $4.00 \pm 0.53^{\mathrm{a}-\mathrm{c}}$ & $4.13 \pm 0.35^{\mathrm{a}-\mathrm{c}}$ & $3.75 \pm 0.71^{\mathrm{a}-\mathrm{c}}$ \\
\hline
\end{tabular}

${ }^{\mathrm{a}} \mathrm{P}<0.05$ vs. control group; ${ }^{\mathrm{b}} \mathrm{P}<0.05$ vs. MPTP group; ${ }^{\mathrm{P}}>0.05$ vs. control group and MPTP + SSTF group. MPTP, 1-methyl-4-phenyl-1,2,3,6-tetrahydropyridine; SSTF, Scutellaria baicalensis stem-leaf total flavonoid. Values are presented as means \pm standard deviations

above the ground. The ability of the mice to grab the cotton with their forepaws was observed, and the hanging time was recorded with a scoring system as follows: $0-4 \mathrm{sec}, 0 ; 5-9 \mathrm{sec}, 1$; 10-14 sec, 2 ; 15-19 sec, 3; 20-24 sec, 4; 25-29 sec, 5; >30 sec, 6.

Histopathological analysis. The mice were euthanized by overdose with chloral hydrate (ShangHai YuanYe Bio-tech Co., Ltd., Shanghai, China) and the midbrain was collected on day nine of the treatment. The brain tissue samples were processed with $4 \%$ polysorbate-phosphate buffered saline fluid and stored at $4^{\circ} \mathrm{C}$ for $24 \mathrm{~h}$. The midbrain tissue samples were sliced into $6-\mu \mathrm{m}$ sections.

Toluidine blue staining was performed with $1 \%$ toluidine blue solution, with incubation at $50-60^{\circ} \mathrm{C}$ for $20-40 \mathrm{~min}$. The brain tissue samples were washed with distilled water 2-3 times and processed with $95 \%$ alcohol for differentiation. Ethanol was used to dehydrate the tissue samples through a gradient and dimethylbenzene (ShangHai YuanYe Bio-tech Co., Ltd.) was used for vitrification. The tissue samples were sealed with neutral gum. The number and morphology of dopaminergic neurons were observed under a high power microscope; dopamine-positive cells were defined as those with blue staining. The number of dopaminergic cells were counted from four visual fields under a high power microscope. For each field, the counts were performed three times and the mean value of the three counts was used.

Serum malondialdehyde (MDA) measurement. Enzyme-linked immunosorbent assay (ELISA; ShangHai YuanYe Bio-tech Co., Ltd.) was performed according to the manufacturer's instructions, to measure the serum levels of MDA. Venous blood $(5 \mathrm{ml})$ was collected from the tail of the mice and centrifuged at $100 \times \mathrm{g}$ for $10 \mathrm{~min}$. The level of MDA was determined at a wavelength of $450 \mathrm{~nm}$ by spectrophotography.

Statistical analysis. All data were presented as the mean \pm standard deviation. SPSS 13.0 statistical software (SPSS, Inc., Chicago, IL, USA) was used to perform statistical analysis. One-way analysis of variance was used to compare differences between groups and $\mathrm{P}<0.05$ was considered to indicate a statistically significant difference.

\section{Results}

Behavior changes. After injecting MPTP, bradykinesia, muscle rigidity, piloerection, hunched postures, and shortened steps were observed. These symptoms gradually eased 15-30 min after the injection. All animals became hypothermic $1 \mathrm{~h}$ after the MPTP injection and, as shown in Table I, the PD symptoms in the MPTP group endured for longer than the MPTP + SSTF $(\mathrm{P}<0.05)$ and SSTF + MPTP $(\mathrm{P}<0.05)$ groups. No statistically significant difference was identified in the behavioral measures between the MPTP + SSTF and SSTF + MPTP groups $(\mathrm{P}>0.05)$. The behavior scores in the MPTP + SSTF and SSTF + MPTP group were lower than in the MPTP group $(\mathrm{P}<0.05)$. In addition, the mean hanging scores in the MPTP + SSTF and SSTF + MPTP groups were higher than those of the MPTP group $(\mathrm{P}<0.05$; Table II).

Serum MDA concentration. The mean serum MDA concentrations in the MPTP + SSTF and SSTF + MPTP groups were similar to the control group $(\mathrm{P}>0.05)$, which were significantly lower than in the MPTP group $(\mathrm{P}<0.05$; Table III). 
Table III. Serum MDA concentration in the four groups.

\begin{tabular}{ll}
\hline Group & MDA, $\mu \mathrm{mol} / 1$ \\
\hline Control & $3.76 \pm 0.17$ \\
MPTP & $5.76 \pm 0.18^{\mathrm{a}}$ \\
MPTP + SSTF & $3.43 \pm 0.16^{\mathrm{a}, \mathrm{b}}$ \\
SSTF + MPTP & $3.40 \pm 0.15^{\mathrm{a}, \mathrm{b}}$ \\
\hline
\end{tabular}

${ }^{\mathrm{a}} \mathrm{P}<0.05$ vs. control group; ${ }^{\mathrm{b}} \mathrm{P}<0.05$ vs. MPTP group. MDA, malondialdehyde; MPTP, 1-methyl-4-phenyl-1,2,3,6-tetrahydropyridine; SSTF, Scutellaria baicalensis stem-leaf total flavonoid. Values are presented as means \pm standard deviations.
Table IV. Number of dopaminergic neurons in the four groups.

\begin{tabular}{lc}
\hline Groups & Dopaminergic cells \\
\hline Control & $95.63 \pm 3.78$ \\
MPTP & $43.75 \pm 2.49^{\mathrm{a}}$ \\
MPTP + SSTF & $65.63 \pm 4.00^{\mathrm{a}, \mathrm{b}}$ \\
SSTF + MPTP & $67.88 \pm 4.36^{\mathrm{a}, \mathrm{b}}$ \\
\hline
\end{tabular}

${ }^{\mathrm{a}} \mathrm{P}<0.05$ vs. control group; ${ }^{\mathrm{b}} \mathrm{P}<0.05$ vs. MPTP group. МРTP, 1-methyl-4-phenyl-1,2,3,6-tetrahydropyridine; SSTF, Scutellaria baicalensis stem-leaf total flavonoid. Values are presented as means \pm standard deviations.

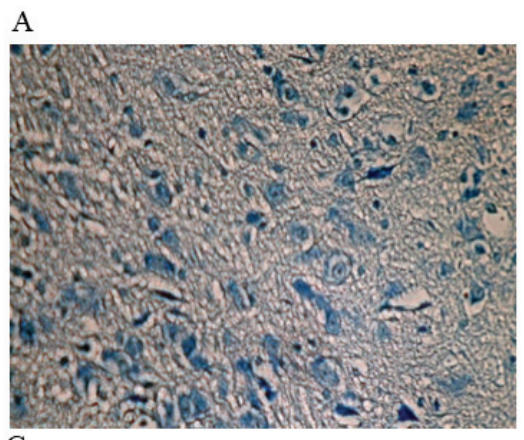

B
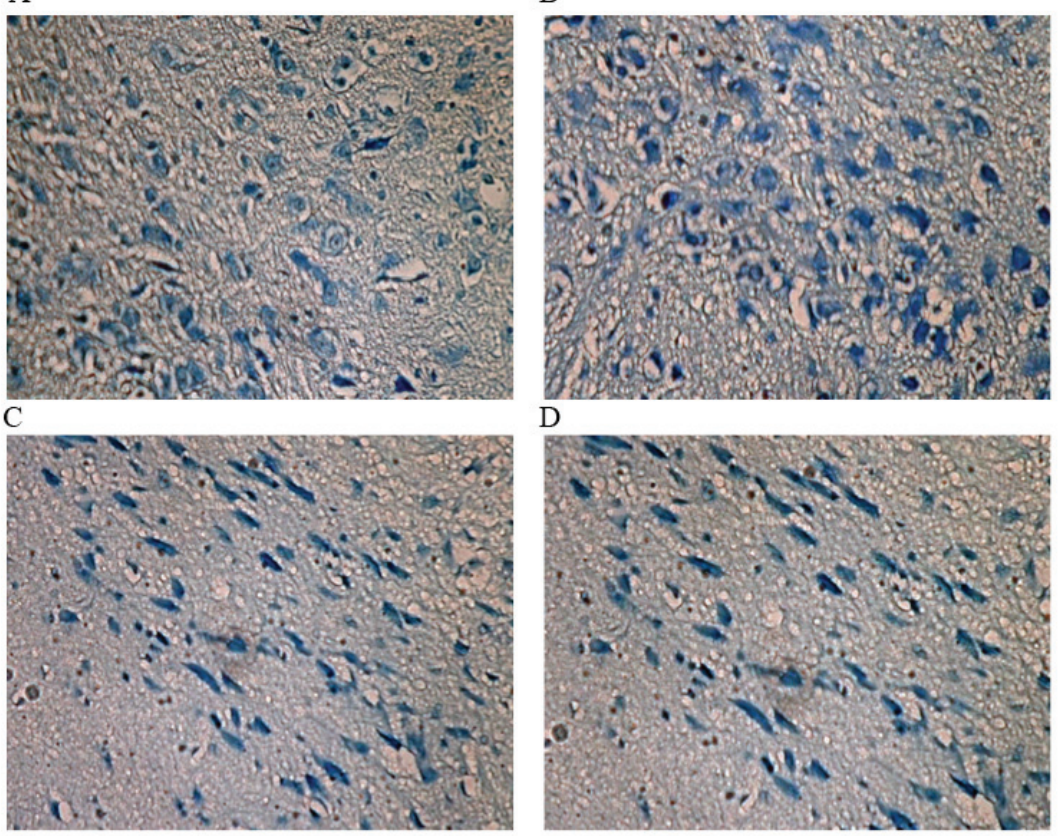

$\mathrm{D}$

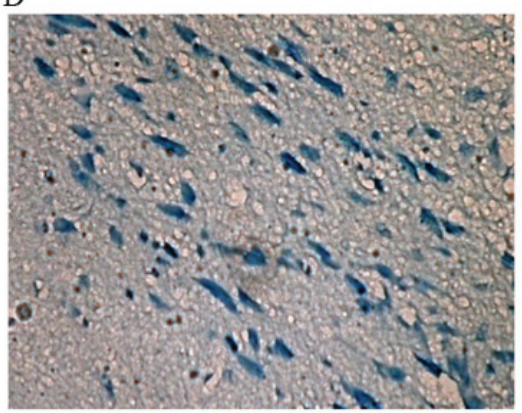

Figure 1. Dopaminergic neurons in the substantia nigra compact (3,3'-diaminobenzidine; magnification, x200). (A) Control group: The number of dopaminergic neurons is greater than in the MPTP group. Morphologically, the neurons are medium in size and cone-shaped, and the cytoplasm is colorless. The nucleolus is small, but clearly visible. (B) SSTF + MPTP group: The number of dopaminergic neurons is the largest (not including the control group). These neurons are medium-sized, cone-shaped, and arranged in ribbons. The neurons demonstrated complete morphology, in which the cytoplasm was hyperchromatic and the nucleolus was clear. Nissl bodies are distributed evenly in the cytoplasm. (C) MPTP group: The number of dopaminergic neurons is clearly markedly compared with the control group. The neurons are clearly atrophied and the nucleoli are smaller. The Nissl bodies are unevenly distributed. (D) MPTP + SSTF group: Compared with the SSTF + MPTP group, the number and morphology of dopaminergic neurons demonstrated no significant difference. MPTP, 1-methyl-4-phenyl-1,2,3,6-tetrahydropyridine; SSTF, Scutellaria baicalensis stem-leaf total flavonoid.

Positive cells in the SNC. In the control group, the number of dopaminergic neurons (Table IV) was significantly greater than in the MPTP group $(\mathrm{P}=0.013)$. The neurons were medium sized and cone-shaped. The color of the cytoplasm was very light and the nucleolus was small, but clearly visible. Of the treatment groups, the SSTF + MPTP group exhibited the largest number of dopaminergic neurons. These neurons were medium-sized, cone-shaped and arranged in ribbons. The neurons demonstrated complete morphology, in which the cytoplasm was hyperchromatic and the nucleolus was clear. Nissl bodies were distributed evenly in the cytoplasm. In the MPTP group, the number of dopaminergic neurons was markedly decreased compared with the control group. The neurons were clearly atrophied, the nucleoli were smaller in size and
Nissl's bodies were unevenly distributed. When compared with the control group, the MPTP + SSTF demonstrated no significant differences in the number and morphology of dopaminergic neurons (Fig. 1).

\section{Discussion}

Scutellaria baicalensis is a medicinal plant widely distributed in Asia. Its dry root, Radix Scutellariae, has been demonstrated to exert free radical scavenging and antioxidant activities, which can be used to treat various types of chronic illness, such as inflammation, allergies, respiratory and cardiovascular disease, and fever (4-6), Baicalein, a flavonoid obtained from Radix Scutellariae provides neuroprotective 
effects for 6-hydroxydopamine-induced animal models of PD $(7,8)$. Baicalein has also been shown to protect against rotenone-induced neurotoxicity in PC12 cells and isolated rat brain mitochondria (9), and to attenuate inflammation-mediated degeneration of dopaminergic neurons (10). Furthermore, in mice models, Baicalein protects against MPTP-induced neurotoxicity (11-13).

In the present study, the effect of SSTF, an extract from the stems and leaves of Scutellaria baicalensis, on MPTP-induced neurological damage in mice was investigated. Treatment with SSTF was associated with reduced behavioral abnormalities and higher hanging scores than the mice in the MPTP only group. The mean number of dopaminergic neurons in the SNC tissue samples of the SSTF groups was higher than in the MPTP only group. These results suggest that 5 days of SSTF treatment is associated with neuroprotective effects against MPTP-induced neurotoxicity.

Various studies indicate that free radical-mediated oxidative damage is involved in the pathogenesis of neurodegenerative disease $(1,4)$. MDA is the product of lipid peroxidation breakdown and its assessment is considered to be a reliable marker of oxidative damage. Patients with PD present with an increased serum level of MDA (14). In the present study, the serum level of MDA was elevated in those mice that were treated with MPTP; however, in mice treated with SSTF, the MDA serum levels were lower than in those without SSTF treatment. These results indicate that SSTF improves the anti-oxidation index and reduces the oxygen free radical damage to the cytomembrane. This action on MDA may have contributed to the neuroprotective effects and behavioral improvement of the mice in the present study.

In conclusion, SSTF appeared to improve behaviors and reduce damage to the dopaminergic neurons of the SNC in a mice model of PD. These beneficial effects may be associated with the inhibition of oxidation, alleviating the damage of oxygen free radicals to dopaminergic neurons. Thus, SSTF, as a plant-estrogen, may be administered to treat PD in future.

\section{Acknowledgements}

The present study was supported, in part, by a grant from the National Natural Science Foundation China (grant no. 30170334).

\section{References}

1. Connolly BS and Lang AE: Pharmacological treatment of Parkinson disease: A review. JAMA 311: 1670-1683, 2014.

2. Song HR, Cheng JJ, Miao H and Shang YZ: Scutellaria flavonoid supplementation reverses ageing-related cognitive impairment and neuronal changes in aged rats. Brain Inj 23: 146-153, 2009.

3. Li LH, Qin HZ, Wang JL, Wang J, Wang XL and Gao GD: Axonal degeneration of nigra-striatum dopaminergic neurons induced by 1-methyl-4-phenyl-1,2,3,6-tetrahydropyridine in mice. J Int Med Res 37: 455-463, 2009.

4. Gao Z, Huang K, Yang X and Xu H: Free radical scavenging and antioxidant activities of flavonoids extracted from the radix of Scutellaria baicalensis Georgi. Biochim Biophys Acta 16: 643-650, 1999.

5. Gong X and Sucher NJ: Stroke therapy in traditional Chinese medicine (TCM): Prospects for drug discovery and development. Trends Pharmacol Sci 20: 191-196, 1999.

6. Kang DG, Yun C and Lee HS: Screening and comparison of antioxidant activity of solvent extracts of herbal medicines used in Korea. J Ethnopharmacol 87: 231-236, 2003.

7. Mu X, He G, Cheng Y, Li X, Xu B and Du G: Baicalein exerts neuroprotective effects in 6-hydroxydopamine-induced experimental parkinsonism in vivo and in vitro. Pharmacol Biochem Behav 92: 642-648, 2009.

8. Yu X, He GR, Sun L, Lan X, Shi LL, Xuan ZH and Du GH: Assessment of the treatment effect of baicalein on a model of Parkinsonian tremor and elucidation of the mechanism. Life Sci 91: 5-13, 2012.

9. Li XX, He GR, Mu X, Xu B, Tian S, Yu X, Meng FR, Xuan ZH and Du GH: Protective effects of baicalein against rotenone-induced neurotoxicity in PC12 cells and isolated rat brain mitochondria. Eur J Pharmacol 674: 227-233, 2012.

10. Li FQ, Wang T, Pei Z, Liu B and Hong JS: Inhibition of microglial activation by the herbal flavonoid baicalein attenuates inflammation-mediated degeneration of dopaminergic neurons. J Neural Transm Vienna 112: 331-347, 2005.

11. Cheng Y, He G, Mu X, Zhang T, Li X, Hu J, Xu B and Du G: Neuroprotective effect of baicalein against MPTP neurotoxicity: Behavioral, biochemical and immunohistochemical profile. Neurosci Lett 441: 16-20, 2008.

12. Mu X, He GR, Yuan X, Li XX and Du GH: Baicalein protects the brain against neuron impairments induced by MPTP in C57BL/6 mice. Pharmacol Biochem Behav 98: 286-291, 2011.

13. Li XZ, Zhang SN, Liu SM and Lu F: Recent advances in herbal medicines treating Parkinson's disease. Fitoterapia 84: 273-285, 2013.

14. Sanyal J, Bandyopadhyay SK, Banerjee TK, Mukherjee SC, Chakraborty DP, Ray BC and Rao VR: Plasma levels of lipid peroxides in patients with Parkinson's disease. Eur Rev Med Pharmacol Sci 13: 129-132, 2009. 